\title{
Carroll structures, null geometry, and conformal isometries
}

\author{
Luca Ciambelli, ${ }^{1,2}$ Robert G. Leigh, ${ }^{2,3}$ Charles Marteau, ${ }^{1}$ and P. Marios Petropoulos ${ }^{1}$ \\ ${ }^{1}$ CPHT, CNRS, Ecole Polytechnique, IP Paris, 91128 Palaiseau Cedex, France* \\ ${ }^{2}$ Perimeter Institute for Theoretical Physics, 31 Caroline Street North, Waterloo, \\ Ontario N2L 2Y5, Canada \\ ${ }^{3}$ Department of Physics, University of Illinois, 1110 West Green Street, Urbana, Illinois 61801, USA
}

(Received 21 June 2019; published 12 August 2019)

\begin{abstract}
We study the concept of Carrollian spacetime starting from its underlying fiber-bundle structure. The latter admits an Ehresmann connection, which enables a natural separation of time and space, preserved by the subset of Carrollian diffeomorphisms. These allow for the definition of Carrollian tensors and the structure at hand provides the designated tools for describing the geometry of null hypersurfaces embedded in Lorentzian manifolds. Using these tools, we investigate the conformal isometries of general Carrollian spacetimes and their relationship with the BMS group.
\end{abstract}

DOI: 10.1103/PhysRevD.100.046010

\section{INTRODUCTION}

The Carroll group was discovered by Lévy-Leblond in 1965 [1] as a dual contraction of the Poincaré group, operating at vanishing rather than infinite velocity of light. The increasing interest in non-Minkowskian spacetimes possessing nonetheless boostlike isometries has led to more systematic studies of Carrollian constructions. Besides the intrinsic value of the latter (along with Newton-Cartan geometry), the resurgence in the area has been sustained by the parallel growth of two distinct albeit related fields of application. The first involves codimension-one null hypersurfaces in Lorentzian, i.e., hyperbolic pseudo-Riemannian, manifolds. The second concerns the development of flat holography.

Carroll structures were introduced in [2-5] as alternatives to Riemannian or Newton-Cartan geometries. According to these authors, Carroll structures consist of a $d+1$-dimensional manifold $\mathcal{C}$ equipped with a degenerate metric $g$ and a vector field $E$, which defines the kernel of the metric, i.e., $g(E,)=$.0 . In this definition, the Carroll group emerges as the isometry group of flat Carrollian structures, whereas general diffeomorphisms are always available. Because of the field $E$, the Carroll structure defines a natural separation between time and space, and a

\footnotetext{
Centre de Physique Théorique, Centre National de la Recherche Scientifique, Institut Polytechnique de Paris.

Published by the American Physical Society under the terms of the Creative Commons Attribution 4.0 International license. Further distribution of this work must maintain attribution to the author(s) and the published article's title, journal citation, and DOI. Funded by SCOAP.
}

subset of diffeomorphisms arises, the Carrollian diffeomorphisms, which preserves this separation.

Given their defining properties, Carroll structures are expected to arise systematically as geometries on null hypersurfaces of relativistic spacetimes, because the induced metric inherited from the embedding is degenerate. ${ }^{1}$ There are several notable instances of null hypersurfaces. Generally, null hypersurfaces occur as components of the boundary of causal diamonds and related structures, relevant in the study of entanglement. One also finds null hypersurfaces in other important physical situations, such as blackhole horizons and the hypersurfaces appearing at lightlike infinity of asymptotically flat spacetimes (commonly designated as $\mathcal{I}^{ \pm}$). The latter makes the bridge with asymptotically flat holography, in which the putative dual degrees of freedom are expected to be defined precisely on this nullinfinity hypersurface. In fact, asymptotically flat holography has been probably the first arena of application of Carrollian physics $[9,10]$, not so much because of the geometric structure the boundary is endowed with (its Carrollian nature was identified much later), but for the emergence of the BMS symmetry. The BMS group was discovered in 1962 by Bondi, van der Burg, Metzner and Sachs [11,12] as the asymptotic isometry group of asymptotically flat spacetimes towards null infinity (see, e.g., $[13,14])$. It was in particular proven [3] that the $\mathfrak{b} \mathfrak{m} \mathfrak{s}(d+2)$ algebra is isomorphic to the conformal Carroll algebra $\mathfrak{c} \mathfrak{a r r}(d+1)$ for $d=1,2$. This is yet another sign corroborating the triangle "Carrollnull-BMS."

The aim of the present work is to revisit this web of relationships and provide an alternative perspective to some of its aspects. Our analysis follows two paths. On the one

\footnotetext{
${ }^{1}$ For related work, see [6-8].
} 
hand, we define a Carrollian spacetime by recasting a Carroll structure in terms of a fiber bundle. ${ }^{2}$ The ingredients this fiber bundle is equipped with are thus an Ehresmann connection, a degenerate metric and a scale factor, ${ }^{3}$ all assumed a priori time and space dependent. This provides us with a geometric understanding of the appearance of Carrollian diffeomorphisms and the reduction of spacetime tensors to Carrollian tensors. Carrollian spacetimes with the above set of ingredients are also naturally revealed in null embedded hypersurfaces. On the other hand, we discuss the conformal isometry algebra of general Carrollian spacetimes. In the shearless case (properly defined shortly), we generally recover the familiar algebra of transformations, which in arbitrary dimension is the semidirect sum of the conformal isometry algebra with supertranslations. In two and three dimensions, this coincides with BMS. The strength of our results resides in their wide validity for shearless but otherwise arbitrary Carrollian spacetimes. In the literature there have been other proposals made for a notion of geometry defined on null embedded hypersurfaces, the "universal structures" (see, e.g., $[14,19]$ ). Different such proposals may lead to different algebras that preserve the given structure, along with a related choice of partial gauge fixing.

\section{CARROLLIAN SPACETIMES AS FIBER BUNDLES}

\section{A. The intrinsic definition}

A $d+1$-dimensional Carrollian spacetime $\mathcal{C}$ is elegantly described in terms of a fiber bundle with one-dimensional fiber and a $d$-dimensional base $\mathcal{S}$ thought of as space, the fiber coordinate being time. As usual, the bundle structure provides a projection $\pi: \mathcal{C} \rightarrow \mathcal{S}$, which defines in turn a surjective linear map between the corresponding tangent bundles, $\mathrm{d} \pi: T \mathcal{C} \rightarrow T \mathcal{S}$. It is convenient to choose a local coordinate system $x=\{t, \mathbf{x}\}$ such that the action of the projector simplifies to $\pi:(t, \mathbf{x}) \rightarrow \mathbf{x}$; that is, $t$ is the fiber coordinate.

One can define a vertical sub-bundle as $V=\operatorname{ker}(\mathrm{d} \pi)$. The above coordinate set has been chosen such that $V$ is given by all sections of $T C$ proportional to $\partial_{t}$ (vectors of the vertical tangent subspace $V_{(t, \mathbf{x})}$ are of the form $\left.W^{t} \partial_{t}\right)$. In order to split the tangent space $T_{(t, \mathbf{x})} \mathcal{C}$ into a direct sum of vertical and horizontal components, $V_{(t, \mathbf{x})} \oplus H_{(t, \mathbf{x})}$ (smooth everywhere, i.e., valid for the tangent bundle, $T C=V \oplus H)$, one needs an Ehresmann connection. With this connection, the linear map $\mathrm{d} \pi$ restricted to $H_{(t, \mathbf{x})}$ sets a one-to-one correspondence between $H_{(t, \mathbf{x})}$ and $T_{\mathbf{x}} \mathcal{S}$. This

\footnotetext{
${ }^{2}$ This concept was introduced earlier in Refs. $[15,16]$ as an "ambient structure."

${ }^{3}$ Note that these ingredients all appear within the context of Carrollian fluids and the fluid-gravity correspondence, as in Refs. $[17,18]$.
}

allows one to lift vertically vectors $W=W^{i} \partial_{i} \in T_{\mathbf{x}} \mathcal{S}$ to $\bar{W}=W^{i} E_{i} \in H_{(t, \mathbf{x})}$, where

$$
E_{i}=\partial_{i}+b_{i}(t, \mathbf{x}) \partial_{t}, \quad i=1, \ldots, d
$$

provide a basis for $H_{(t, \mathbf{x})}$. The Ehresmann connection is encoded in the one-form $\boldsymbol{b}=b_{i}(t, \mathbf{x}) \mathrm{d} x^{i} \in T^{*} \mathcal{C}$.

The Ehresmann connection has many facets. On the one hand, it provides a lift of curves in $\mathcal{S}$ onto curves in $\mathcal{C}$ such that the tangent vectors to the latter are horizontal. On the other hand, it makes it possible to realize the splitting $T C=V \oplus H$ through the definition of the projector $p$ acting on $T C$ with image $V$ and kernel $H$,

$$
p=\partial_{t} \otimes\left(\mathrm{d} t-b_{i}(t, \mathbf{x}) \mathrm{d} x^{i}\right)
$$

We call the fiber bundle $\mathcal{C}$ a Carrollian spacetime, once endowed with a degenerate metric $g$ whose onedimensional kernel coincides with the vertical subbundle $V$,

$$
g(X, .)=0, \quad \forall X \in V .
$$

In the local coordinate system this imposes the metric to be of the form

$$
g=g_{i j}(t, \mathbf{x}) \mathrm{d} x^{i} \otimes \mathrm{d} x^{j},
$$

providing a time-dependent notion of distances.

At this point of the presentation, it is worth mentioning that the triple $(\mathcal{C}, V, g)$ corresponds to the definition of a weak Carrollian structure given in [4]. From the spacetime viewpoint, the fiber-bundle structure and the accompanying Ehresmann connection are the key ingredients for the intrinsic horizontal versus vertical splitting of the tangent bundle, and more generally of any tensor bundle.

The coordinate system $\{t, \mathbf{x}\}$ is adapted to the splitting at hand, as is any new chart obtained through the transformation

$$
t \mapsto t^{\prime}(t, \mathbf{x}) \quad \text { and } \quad \mathbf{x} \mapsto \mathbf{x}^{\prime}(\mathbf{x})
$$

The motivation for introducing the fiber-bundle structure is, among others, to make these diffeomorphisms natural, being a reparametrization of the fiber coordinate at each spatial point and a change of coordinates on the base, respectively. With this, the Jacobian matrix $J_{\nu}^{\mu}=\frac{\partial x^{\prime \mu}}{\partial x^{\nu}}$ is upper triangular,

$$
\left(\begin{array}{cc}
J(t, \mathbf{x}) & J_{i}(t, \mathbf{x}) \\
0 & J_{i}^{j}(\mathbf{x})
\end{array}\right),
$$

since 
$\mathrm{d} t^{\prime}=J(t, \mathbf{x}) \mathrm{d} t+J_{i}(t, \mathbf{x}) \mathrm{d} x^{i}, \quad \mathrm{~d} x^{j}=J_{i}^{j}(\mathbf{x}) \mathrm{d} x^{i}$,

or equivalently

$$
\partial_{t}=J(t, \mathbf{x}) \partial_{t}^{\prime}, \quad \partial_{i}=J_{i}(t, \mathbf{x}) \partial_{t}^{\prime}+J_{i}^{j}(\mathbf{x}) \partial_{j}^{\prime} .
$$

These diffeomorphisms were called Carrollian in [17]. Every spacetime tensor field can be decomposed intrinsically into vertical and horizontal components, the latter transforming tensorially under Carrollian diffeomorphisms. These components are the Carrollian tensors introduced in [17]. An example of a Carrollian tensor is the metric (4), whose components transform as

$$
g_{i j}^{\prime}=J^{-1 k}{ }_{i} J^{-1 \ell}{ }_{j} g_{k \ell},
$$

i.e., as a rank- $(0,2)$ Carrollian tensor field. In order to maintain the invariance of $p$ in Eq. (2), the components of the Ehresmann connection must transform as

$$
b_{j}^{\prime}=J_{j}^{-1 i}\left(J b_{i}+J_{i}\right) .
$$

For reasons that become clear in the course of the paper, it is convenient to introduce a density $\Omega(t, \mathbf{x})$, transforming under Carrollian diffeomorphisms as ${ }^{4}$

$$
\Omega^{\prime}\left(t^{\prime}, \mathbf{x}^{\prime}\right)=J(t, \mathbf{x})^{-1} \Omega(t, \mathbf{x}) .
$$

With this density, one defines a new basis vector of $V_{(t, \mathbf{x})}$ as

$$
E=\Omega(t, \mathbf{x})^{-1} \partial_{t}
$$

Together with the $H_{(t, \mathbf{x})}$ basis vectors $E_{i}$ defined in (1), we obtain a frame $E_{\mu}, \mu=0, \ldots, d$, adapted to the split tangent space and transforming canonically under Carrollian diffeomorphisms $\left(E_{0} \equiv E\right)$,

$$
E^{\prime}=E \quad \text { and } \quad E_{i}^{\prime}=J^{-1 j}{ }_{i} E_{j} .
$$

The dual coframe, generically referred to as $\boldsymbol{e}^{\mu}, \mu=0, \ldots, d$, is $\left(\boldsymbol{e}^{0} \equiv \boldsymbol{e}\right)$,

$\boldsymbol{e}=\Omega\left(\mathrm{d} t-b_{j} \mathrm{~d} x^{j}\right) \quad$ and $\quad \boldsymbol{e}^{i}=\mathrm{d} x^{i}, \quad i=1, \ldots, d$,

with $\boldsymbol{e}^{i}$ transforming as in (7) and $\boldsymbol{e}^{\prime}=\boldsymbol{e}$.

Any vector $W \in T C$ is decomposed in the above frame as $W=W^{0}(t, \mathbf{x}) E+W^{i}(t, \mathbf{x}) E_{i}$, while any form $\omega \in T^{*} \mathcal{C}$ is $\boldsymbol{\omega}=\omega_{0}(t, \mathbf{x}) \boldsymbol{e}+\omega_{i}(t, \mathbf{x}) \boldsymbol{e}^{i}$. In this basis, the vertical and horizontal components are reduced, i.e., do not mix under Carrollian diffeomorphisms. The vertical components

\footnotetext{
${ }^{4}$ Observe that $\boldsymbol{b}$ transforms as a Carrollian connection density. Strictly speaking, the Ehresmann connection is thus $\boldsymbol{b} \Omega$. To avoid confusion, we should mention that the latter combination was referred to as $\boldsymbol{b}$ in $[17,18]$.
}

remain invariant, while the horizontal transform tensorially under Carroll diffeomorphisms,

$$
W^{\prime 0}=W^{0}, \quad W^{\prime i}=J_{j}^{i} W^{j}, \quad \omega_{0}^{\prime}=\omega_{0}, \quad \omega_{i}^{\prime}=J^{-1 j}{ }_{i} \omega_{j} .
$$

From the horizontal perspective $W^{0}$ and $\omega_{0}$ are scalars, and we refer to them as Carrollian scalars, whereas $W^{i}$ and $\omega_{i}$ are components of a Carrollian vector and a Carrollian one-form. The same reduction properties are valid for rank- $(r, s)$ tensor fields in $T^{(r, s)} \mathcal{C}$. Notice that one can use $g_{i j}=g\left(E_{i}, E_{j}\right)$ and its inverse $g^{i j}$ for lowering and raising spatial indices $i, j, \ldots$ amongst Carrollian tensors.

In terms of the frame (1) and (12), and the coframe (14), the action of the exterior derivative on the generic one-form $\omega$ reads

$$
\mathrm{d} \boldsymbol{\omega}=\left(E\left(\omega_{i}\right)-E_{i}\left(\omega_{0}\right)\right) \boldsymbol{e} \wedge \boldsymbol{e}^{i}+E_{k}\left(\omega_{i}\right) \boldsymbol{e}^{k} \wedge \boldsymbol{e}^{i} .
$$

One can define the Ehresmann curvature as

$$
\mathrm{d} \boldsymbol{e}=\boldsymbol{\varphi} \wedge \boldsymbol{e}+\varpi=\varphi_{i} \boldsymbol{e}^{i} \wedge \boldsymbol{e}+\frac{1}{2} \varpi_{i j} \boldsymbol{e}^{i} \wedge \boldsymbol{e}^{j},
$$

which exhibits a pair of genuine Carrollian tensors. The purely horizontal piece $\boldsymbol{\varpi}$ is a Carrollian two-form, which we call the Carrollian torsion. ${ }^{5}$ It has components

$$
\varpi_{i j}=-\Omega\left(E_{i}\left(b_{j}\right)-E_{j}\left(b_{i}\right)\right) .
$$

The vertical-horizontal mixed components

$$
\varphi_{i}=\Omega E\left(b_{i}\right)+E_{i}(\ln \Omega)
$$

define a Carrollian one-form $\boldsymbol{\varphi}$, the acceleration. Both appear in the Lie bracket of the basis vectors,

$$
\left[E_{i}, E_{j}\right]=-\varpi_{i j} E, \quad\left[E_{i}, E\right]=-\varphi_{i} E,
$$

which is dual to (17).

A natural question to ask is whether $H$ can be thought of as the tangent bundle of codimension-one hypersurfaces in $\mathcal{C}$. If this holds, $\mathcal{C}$ is foliated by a family of hypersurfaces modeled on $\mathcal{S}$. This is indeed possible whenever $H$ is an integrable distribution in $T C$. The corresponding integrability condition originates from Fröbenius' theorem stating that the Lie bracket of horizontal vectors must be horizontal, or equivalently, that the vorticity of the normal (vertical) vector should vanish, $\varpi_{i j}=0$. In other words, the Ehresmann curvature should have no horizontal component.

Besides the above Carrollian tensors emanating from the Ehresmann connection, others can be defined using the

\footnotetext{
${ }^{5}$ The quantity $-\frac{1}{2} \varpi_{i j}$ is also referred to as the Carrollian vorticity of the vector field $E$ [17].
} 
metric $g$. Using time derivatives of the metric (the metric components are generically functions of both $t$ and $\mathbf{x}$ ), we define

$\theta=E(\ln \sqrt{\operatorname{det} g})=\frac{1}{2} g^{i k} E\left(g_{k i}\right), \quad \zeta_{i j}=\frac{1}{2} E\left(g_{i j}\right)-\frac{1}{d} g_{i j} \theta$,

referred to as expansion and shear ( $g^{i j}$ are the components of the inverse of $g$ ). They are respectively a Carrollian scalar and a Carrollian symmetric and traceless rank-two tensor. The latter vanishes if and only if the time dependence in the metric is factorized, $g_{i j}(t, \mathbf{x})=\mathrm{e}^{2 \sigma(t, \mathbf{x})} \tilde{g}_{i j}(\mathbf{x})$, in which case the expansion reads $\theta=d E(\sigma)$. This instance turns out to play a significant role later in the discussion of BMS symmetry (Sec. III).

We can also introduce a connection which defines a horizontal parallel transport for horizontal tensors, i.e., a covariant derivative acting on Carrollian tensors and producing new Carrollian tensors. It was introduced in [17] as $\mathrm{D}=\mathrm{E}+\gamma$, called the Levi-Civita-Carroll connection, with $\gamma$ being the Christoffel-Carroll symbols,

$\gamma_{j k}^{i}(t, \mathbf{x})=\frac{1}{2} g^{i l}\left(E_{j}\left(g_{l k}\right)+E_{k}\left(g_{l j}\right)-E_{l}\left(g_{j k}\right)\right)=\Gamma_{j k}^{i}+c_{j k}^{i}$,

where $\Gamma_{j k}^{i}$ are the ordinary Christoffel symbols and

$c_{j k}^{i}(t, \mathbf{x})=\frac{\Omega}{2} g^{i l}\left(b_{j} E\left(g_{l k}\right)+b_{k} E\left(g_{l j}\right)-b_{l} E\left(g_{j k}\right)\right)$.

This connection, also cast as $\mathrm{D}=\nabla+\mathrm{c}$ with $\nabla$ being the Levi-Civita connection, is metric compatible $\left(D_{k} g_{i j}=0\right)$, and satisfies $\gamma_{[j k]}^{i}=0$. Its curvature tensors can be worked out following [17]. As opposed to the ordinary Levi-Civita connection for Riemannian manifolds, the Levi-CivitaCarroll connection is not the unique metric-compatible and torsionless connection one can define on $T \mathcal{C}$. This question has been examined, e.g., in $[15,16]$.

In the spirit of [2-4], one can introduce the concept of flat Carrollian spacetime given in an adapted coordinate system by

$$
g_{i j}=\delta_{i j}, \quad \Omega=1, \quad b_{i}=\text { const. }
$$

For this case, the Ehresmann curvature $\varpi_{i j}$ as well as the acceleration $\varphi_{i}$, the shear $\zeta_{i j}$ and the expansion $\theta$ vanish, as do the Christoffel-Carroll symbols written above. Carrollian flatness implies that the Ehresmann connection is a pure gauge.

\section{B. Realization on null hypersurfaces}

We discuss now the appearance of the above structures on null hypersurfaces $\mathcal{C}$ of a Lorentzian spacetime $\mathcal{M}$. The pullback $g$ of the ambient metric on null hypersurfaces is degenerate with one-dimensional tangent sub-bundle kernel $V$, and from this perspective the Carrollian structure encompassed in the triple $(\mathcal{C}, V, g)$ emerges naturally. This feature has been discussed by several authors, the more complete account being in the already quoted Refs. $[7,8]$. The fiber bundle with Ehresmann connection approach, which is designed for separating explicitly Carrollian time and space, emerges naturally in null embeddings. This requires appropriate gauge fixing in the ambient Lorentzian spacetime. ${ }^{6}$

We illustrate the above in the case of a $d+2$-dimensional spacetime $\mathcal{M}$ foliated with null hypersurfaces. In this case the ambient metric reads

$$
\begin{aligned}
\mathrm{d} s_{\mathcal{M}}^{2}= & g_{a b} \mathrm{~d} x^{a} \mathrm{~d} x^{b}=-2 \Omega \Xi\left(\mathrm{d} t-b_{i} \mathrm{~d} x^{i}+\theta^{t} \mathrm{~d} r-b_{i} \theta^{i} \mathrm{~d} r\right) \mathrm{d} r \\
& +g_{i j}\left(\mathrm{~d} x^{i}+\theta^{i} \mathrm{~d} r\right)\left(\mathrm{d} x^{j}+\theta^{j} \mathrm{~d} r\right),
\end{aligned}
$$

where $\Omega, \Xi, b_{i}, \theta^{t}, \theta^{i}$ and $g_{i j}$ depend on all the coordinates $(r, t, \mathbf{x})$ and $t$ is a retarded time. The constant- $r$ leaves of the foliation $\mathcal{C}_{r}$ define $d+1$-dimensional null hypersurfaces because the pullback of the metric, $g_{r}=g_{i j}(r, t, \mathbf{x}) \mathrm{d} x^{i} \mathrm{~d} x^{j}$, is indeed degenerate. The diffeomorphisms that preserve the form of this metric are

$r \mapsto r^{\prime}(r), \quad t \mapsto t^{\prime}(r, t, \mathbf{x}), \quad \mathbf{x} \mapsto \mathbf{x}^{\prime}(r, \mathbf{x})$

Defining as usual

$$
J_{b}^{a}=\frac{\partial x^{\prime a}}{\partial x^{b}},
$$

the various quantities involved transform as

$$
\begin{gathered}
\Omega^{\prime}=\left(J_{t}^{t}\right)^{-1} \Omega, \\
b_{j}^{\prime}=J^{-1 i}{ }_{j}\left(J_{t}^{t} b_{i}+J_{i}^{t}\right), \\
g_{i j}^{\prime}=J^{-1 k}{ }_{i} J^{-1 \ell}{ }_{j} g_{k \ell}, \\
\Xi^{\prime}=\left(J_{r}^{r}\right)^{-1} \Xi, \\
\theta^{\prime t}=\left(J_{r}^{t}\right)^{-1}\left(J_{t}^{t} \theta^{t}-J_{r}^{t}+J_{i}^{t} \theta^{i}\right), \\
\theta^{i}=\left(J_{r}^{t}\right)^{-1}\left(J_{j}^{i} \theta^{j}-J_{r}^{i}\right) .
\end{gathered}
$$

Therefore, we see that $\Omega, b_{i}$ and $g_{i j}$ transform on every leaf as they do on a Carrollian spacetime, Eqs. (11), (10), and (9).

\footnotetext{
${ }^{6}$ See [20] for a recent discussion on foliations and symmetries that preserve them.
} 
Hence, the diffeomorphisms (26) are interpreted as Carroll diffeomorphisms on each leaf $\mathcal{C}_{r}$. The other elements $\Xi, \theta^{t}$ and $\theta^{i}$ were not present in the intrinsic definition of the previous section. This is not surprising as they account for the nontrivial $r$-dependence of the residual gauge symmetry (26). For simplicity we fix locally $\Xi=1$ and $\theta^{t}=\theta^{i}=0$. This is achievable using (and therefore fixing) the $r$-dependence of the diffeomorphism (26). Henceforth the bulk metric simplifies to

$$
\mathrm{d} s_{\mathcal{M}}^{2}=-2 \Omega\left(\mathrm{d} t-b_{i} \mathrm{~d} x^{i}\right) \mathrm{d} r+g_{i j} \mathrm{~d} x^{i} \mathrm{~d} x^{j},
$$

with the residual gauge freedom (5),

$$
r \mapsto r, \quad t \mapsto t^{\prime}(t, \mathbf{x}), \quad \mathbf{x} \mapsto \mathbf{x}^{\prime}(\mathbf{x}) .
$$

Indeed, if we were to describe a single null hypersurface, it would also be natural to set $\Xi=1$, and $\theta^{i}$ and $\theta^{t}$ to 0 in its neighborhood. Under the coordinate change (35), $g_{i j}, b_{i}$, and $\Omega$ still transform according to (11), (10), and (9). One can show that $\mathcal{C}_{r}$ equipped with these data is a $d+1$ dimensional Carrollian spacetime, in the lines we discussed earlier. For this we need to exhibit the Ehresmann connection.

The ambient metric (25) allows one to define two independent null vector fields, ${ }^{7}$ sections of $T \mathcal{M}$,

$$
\ell=\frac{1}{\Omega} \partial_{t}, \quad n=\partial_{r}, \quad \ell \cdot n=1 .
$$

The corresponding forms in $T^{*} \mathcal{M}$ are

$$
\boldsymbol{\ell}=-\mathrm{d} r, \quad \boldsymbol{n}=\Omega\left(\mathrm{d} t-b_{i} \mathrm{~d} x^{i}\right) .
$$

Hence, the vector field $\ell$ is normal to $\mathcal{C}_{r}$. Since it is null, it is also tangent to $\mathcal{C}_{r}$ and belongs therefore to $T \mathcal{C}_{r}$. Being the kernel of the degenerate metric $g_{r}$ on $\mathcal{C}_{r}$, it spans the vertical sub-bundle $V_{r}$. The horizontal sub-bundle $H_{r}$ is given by the set of vectors $X$ in $T \mathcal{C}_{r}$ that are orthogonal to $n$,

$$
X \cdot n=0
$$

but since $X \in H_{r}$, by definition

$$
X \cdot \ell=0 .
$$

Thus, writing $X=X^{r} \partial_{r}+X^{t} \partial_{t}+X^{i} \partial_{i}$, Eqs. (38) and (39) lead to $X^{r}=0$ and $X^{t}-b_{i} X^{i}=0$, so that

$$
X \in H_{r} \Leftrightarrow X=X^{i}\left(\partial_{i}+b_{i} \partial_{t}\right)=X^{i} E_{i} .
$$

Consequently, the field $b_{i}(r, t, \mathbf{x})$ plays the role of an Ehresmann connection for each null leaf $\mathcal{C}_{r}$, as one could have anticipated. Notice also that the tensor $p^{a}{ }_{b}=\ell^{a} n_{b}$

\footnotetext{
${ }^{7}$ Our choice of gauge fixing differs from other works as [7,21].
}

has nonzero components $p^{t}{ }_{t}$ and $p_{i}{ }_{i}$. These define a Carrollian tensor, which is the vertical Ehresmann projector $p$ introduced in (2).

Given the above embedding of null hypersurfaces $\mathcal{C}_{r}$, we can determine their extrinsic geometry. This is generally captured by three quantities, the surface gravity, the deformation tensor, and the twist, all built with the projector onto $H_{r} \subset T \mathcal{C}_{r}$,

$$
h_{b}^{a}=\delta_{b}^{a}-n^{a} \ell_{b}-\ell^{a} n_{b} .
$$

Lowering an index we find that the nonzero components are $h_{i j}=g_{i j}(r, t, \mathbf{x})$ and the surface gravity vanishes with our choice of $\ell$. The other extrinsic quantities are respectively given by

$$
\begin{aligned}
D^{a b} & =\frac{1}{2} h^{a c} h^{b d} \mathcal{L}_{\ell} h_{c d}, \\
\omega_{a} & =h_{a}^{b} n_{c} \nabla_{b} \ell^{c},
\end{aligned}
$$

where $\nabla_{a}$ stands for the Levi-Civita connection of $g_{a b}$. In addition, the deformation tensor is reduced to the expansion and the shear

$$
\begin{aligned}
\Theta & =h_{a b} D^{a b}=\frac{1}{2} h^{a b} \mathcal{L}_{\ell} h_{a b}, \\
\sigma^{a b} & =D^{a b}-\frac{\Theta}{d} h^{a b} .
\end{aligned}
$$

For the geometry at hand, the nonvanishing components of the extrinsic tensors, at every $r$, coincide with the Carrollian tensors defined on $\mathcal{C}_{r}$ [see (19) and (21)],

$$
\begin{aligned}
\omega_{i} & =-\frac{1}{2} \partial_{t} b_{i}-\frac{1}{2 \Omega}\left(\partial_{i} \Omega+b_{i} \partial_{t} \Omega\right)=-\frac{1}{2} \varphi_{i}, \\
\Theta & =\frac{1}{\Omega} \partial_{t} \ln \sqrt{g}=\theta, \\
\sigma_{i j} & =\frac{1}{2 \Omega} \partial_{t} g_{i j}-\frac{\Theta}{d} g_{i j}=\zeta_{i j} .
\end{aligned}
$$

The reduced bulk covariance (26), which preserves the form (25), corresponds precisely to the Carrollian diffeomorphisms (5), for which these objects are genuine tensors.

In conclusion, before we turn to the investigation of conformal isometries, the message is that the definition of Carrollian spacetimes as fiber bundles with Ehresmann connection and a degenerate metric is adapted to the description of families of embedded null hypersurfaces where, on any leaf, the induced geometry is Carrollian.

\section{CONFORMAL CARROLLIAN ISOMETRIES}

Carrollian spacetimes $\mathcal{C}$ have been introduced in Sec. II irrespective of any isometry properties. Carrollian diffeomorphisms are not isometries. They are a subgroup of the full diffeomorphism group, compatible with the intrinsic 
splitting in vertical versus horizontal components of the tangent bundle $T \mathcal{C}$, made possible thanks to the Ehresmann connection. The Carroll group emerges precisely on the tangent space at a point. Suppose indeed that we trade the $H$ basis vectors $E_{i}$ for a set of vectors $\hat{E}_{\hat{\imath}}$, orthonormal with respect to $g: g\left(\hat{E}_{\hat{\imath}}, \hat{E}_{\hat{\jmath}}\right)=\delta_{\hat{\imath} \hat{\jmath}}$. The tangent space is now everywhere spanned by $\left\{E, \hat{E}_{\hat{\imath}}, \hat{\imath}=1, \ldots, d\right\}$, whereas for the cotangent space the basis is $\left\{\boldsymbol{e}, \hat{\boldsymbol{e}}^{\hat{\imath}}, \hat{\imath}=1, \ldots, d\right\}$ with $\hat{\boldsymbol{e}}^{\hat{\imath}}\left(\hat{E}_{\hat{\jmath}}\right)=\delta_{\hat{\jmath}}^{\hat{\imath}}$. Automorphisms of the tangent space preserving the vertical vector field $E$ and the orthonormal nature of the $H$ basis are generally as follows:

$$
\left(\begin{array}{ll}
E^{\prime} & \hat{E}_{\hat{\imath}}^{\prime}
\end{array}\right)=\left(\begin{array}{ll}
E & \hat{E}_{\hat{\jmath}}
\end{array}\right)\left(\begin{array}{cc}
1 & B_{\hat{k}} R_{\hat{\imath}}^{\hat{k}} \\
0 & R_{\hat{\imath}}^{\hat{\jmath}_{\hat{\imath}}}
\end{array}\right),
$$

with $R_{\hat{\imath}}^{\hat{k}}(t, \mathbf{x})$ being the elements of a $d$-dimensional orthogonal matrix and $B_{\hat{k}}(t, \mathbf{x}), d$ numbers. The explicit dependence on the coordinates underlines that this transformation need not be the same at every point of $\mathcal{C}$. These transformations are the $d+1$-dimensional Carroll boosts (the full Carroll group also includes spacetime translations). They rotate the horizontal frame and coframe, and produce a rotation plus a shift proportional to $B$ on the Ehresmann connection. This latter statement can be made explicit by writing

$$
\hat{E}_{\hat{\imath}}=E_{\hat{\imath}}^{j} \partial_{j}+b_{\hat{\imath}} \partial_{t}
$$

the transformation (45) thus implies

$$
E^{\prime j}{ }_{\hat{\imath}}=E^{j}{ }_{\hat{k}} R_{\hat{\imath}}^{\hat{k}} \quad \text { and } \quad b_{\hat{\imath}}^{\prime}=\left(b_{\hat{k}}+\Omega^{-1} B_{\hat{k}}\right) R_{\hat{\imath}}^{\hat{k}_{\hat{\imath}}} .
$$

The Carroll boosts play for the tangent bundle of a Carrollian spacetime the same role as the Lorentz group does for the tangent bundle of a pseudo-Riemannian manifold.

The Carroll group appears also as the isometry group of the flat Carroll manifold introduced in Eqs. (24). These isometries are diffeomorphisms generated by vectors $\xi$ such that $\mathcal{L}_{\xi} g=0, \mathcal{L}_{\xi} E=0$, and shifting the Ehresmann connection by an arbitrary constant. One finds

$$
\xi^{0}=\beta_{j} x^{j}+\gamma, \quad \xi^{i}=\omega^{i}{ }_{j} x^{j}+\epsilon^{i},
$$

with all entries constant and $\omega_{k j}=\delta_{k i} \omega^{i}{ }_{j}$ antisymmetric. These are precisely the $(d+2)(d+1) / 2$ generators of the Carroll algebra $\mathfrak{c a r r}(d+1)$.

We would like to enter now the core of our discussion about conformal Carrollian isometries for generic Carrollian spacetimes. We first define them, and then solve the associated differential equations under the assumption of the absence of shear. This enables us to exhibit a rather universal algebra, which gives a generalized version of the infinitedimensional conformal Carroll algebra $\mathfrak{c c a r r}(d+1)$.
We define Carrollian conformal Killing vector fields $\xi$ by imposing

$$
\mathcal{L}_{\xi} g=\lambda g
$$

where $\lambda(t, \mathbf{x})$ is an a priori arbitrary function. Setting $\xi=f(t, \mathbf{x}) E+\xi^{i}(t, \mathbf{x}) E_{i}$ we obtain

$$
\begin{aligned}
\mathcal{L}_{\xi} g= & \left(2 g_{i j} \partial_{t} \xi^{i}\right) \mathrm{d} t \mathrm{~d} x^{j}+\left(\left(\Omega^{-1} f+b_{k} \xi^{k}\right) \partial_{t} g_{i j}\right. \\
& \left.+\xi^{k} \partial_{k} g_{i j}+g_{i k} \partial_{j} \xi^{k}+g_{j k} \partial_{j} \xi^{k}\right) \mathrm{d} x^{i} \mathrm{~d} x^{j} \\
= & \left(2 \Omega^{-1} g_{i j} \partial_{t} \xi^{i}\right) \boldsymbol{e} \boldsymbol{e}^{j}+\left(2 f\left(\zeta_{i j}+\frac{1}{d} \theta g_{i j}\right)\right. \\
& \left.+D_{i} \xi_{j}+D_{j} \xi_{i}\right) \boldsymbol{e}^{i} \boldsymbol{e}^{j},
\end{aligned}
$$

where $D_{i}$ stands for the Levi-Civita-Carroll connection introduced in (22). Observe that the time dependence of the metric enters these expressions explicitly and one might expect it to alter significantly the structure of the conformal isometry algebra. At the same time one should also stress that in the absence of time dependence, neither the Ehresmann connection nor the scale factor $\Omega(t, \mathbf{x})$ plays a role in the analysis of conformal properties, which would reduce to the analysis in $[2-4]{ }^{8}$ The first term of (51) translates through Eq. (49) into

$$
\partial_{t} \xi^{i}(t, \mathbf{x})=0 .
$$

This imposes that $\xi$ is the generator of a Carrollian diffeomorphism [it ensures the vanishing entry in (6) since it imposes $\left.\xi^{i}(t, \mathbf{x})=Y^{i}(\mathbf{x})\right]$, and this is assumed systematically here. Hence the core of the definition of conformal Carrollian isometries is in the second term of (51), leading to

$$
2 f\left(\zeta_{i j}+\frac{1}{d} \theta g_{i j}\right)+D_{i} Y_{j}+D_{j} Y_{i}=\lambda g_{i j}
$$

The trace of this equation determines $\lambda$,

$$
\lambda(t, \mathbf{x})=\frac{2}{d}\left(f \theta+D_{i} Y^{i}\right)(t, \mathbf{x}),
$$

and substitution back into (53) then gives

$$
D_{i} Y_{j}+D_{j} Y_{i}-\frac{2}{d} D_{k} Y^{k} g_{i j}=-2 f \zeta_{i j}
$$

At the present stage, the equations to be solved for finding the components of the conformal Killing vectors $f(t, \mathbf{x})$ and $Y^{i}(\mathbf{x})$ are Eqs. (55), which are a set

\footnotetext{
${ }^{8}$ Notice that $\xi=\left(\Omega^{-1} f+b_{k} \xi^{k}\right) \partial_{t}+\xi^{i} \partial_{i}$. Equation (50) depends on $b_{k}$ and $\Omega$ only through $\xi^{t} \equiv \Omega^{-1} f+b_{k} \xi^{k}$.
} 
of time-dependent partial differential equations sourced by the Carrollian shear.

In the Carrollian case under consideration, as a consequence of the degenerate nature of the metric, this set-in other words Eq. (49)—is not sufficient for defining conformal Killing fields. In order to proceed, we must refine our definition of the latter. We further impose vanishing shear for the Carrollian spacetime, and with this the full conformal algebra can be unraveled without any further restriction on the Carrollian data $g_{i j}, \Omega$, and $b_{i}$, generalizing thereby the range of validity of the results obtained in [2-4].

We note that for $\xi=f(t, \mathbf{x}) E+Y^{i}(\mathbf{x}) E_{i}$, the Lie derivative of the vertical vector field $E$ is itself vertical, satisfying

$$
\mathcal{L}_{\xi} E=\mu E,
$$

where

$$
\mu(t, \mathbf{x})=-E(f)-\varphi_{i} Y^{i}
$$

A precise definition of the conformal Carrollian Killing vectors is reached by setting a relation among the $a$ priori independent functions $\lambda(t, \mathbf{x})$ and $\mu(t, \mathbf{x})$. The guideline for this is Weyl covariance, because a desirable feature for conformal Killing fields is their insensitivity to Weyl rescalings of the metric.

We define Weyl rescalings as $g \mapsto g / \mathcal{B}(t, \mathbf{x})^{2}$ and $\boldsymbol{b}$ invariant [this is required for the spatial vectors $E_{i}$ in (1) to remain well defined], supplemented with $\Omega(t, \mathbf{x}) \mapsto$ $\mathcal{B}(t, \mathbf{x})^{-z} \boldsymbol{\Omega}(t, \mathbf{x})$ for some real number $z$, the dynamical exponent. Under such rescalings, $\xi$ has Weyl weight 0 , which implies that $Y^{i}$ and $f$ have weights 0 and $-z$. Therefore, $\lambda(t, \mathbf{x})$ and $\mu(t, \mathbf{x})$ transform as

$$
\lambda \mapsto \lambda-2 Y^{i} E_{i}(\ln \mathcal{B}), \quad \mu \mapsto \mu+z Y^{i} E_{i}(\ln \mathcal{B})
$$

Thus, the combination $2 \mu+z \lambda$ is Weyl covariant (actually invariant). Setting it to 0 ,

$$
2 \mu(t, \mathbf{x})+z \lambda(t, \mathbf{x})=0,
$$

is compatible with the basic expected attributes of Killing vectors, as stressed earlier.

Equations (49) and (59) define our conformal Killing fields. It should be mentioned that (59) was introduced in [2-4] with $z=-2 / N$ and $N$ being a positive integer, following the requirement that $\mathcal{L}_{\xi}\left(g \otimes E^{\otimes N}\right)=0$. Leaving $z$ arbitrary does not support such a geometrical interpretation, but is nonetheless consistent. The case $z=1$ (i.e., $N=2$ ), where time and space equally dilate, pertains when the Carrollian spacetime emerges on an embedded null hypersurface in a pseudo-Riemannian geometry.
The combination of (54), (57), and (59) leads to 9

$$
D_{i} Y^{i}-\frac{d}{z} \varphi_{i} Y^{i}-\frac{d}{z}\left(E(f)-\frac{z}{d} \theta f\right)=0 .
$$

Summarizing, the conformal isometry group as defined in (49) and (59) for a Carrollian spacetime described in terms of $\Omega(t, \mathbf{x}), b_{i}(t, \mathbf{x})$, and $g_{i j}(t, \mathbf{x})$ is the set of solutions $f(t, \mathbf{x})$ and $Y^{i}(\mathbf{x})$ of Eqs. (55) and (60) for a given choice of $z$.

At this point we restrict our analysis to Carroll spacetimes with vanishing shear, $\zeta_{i j}=0$, because in this case the system (55) and (60) can be solved. As stated previously, $\zeta_{i j}$ vanishes if and only if the time dependence of the metric is conformal,

$$
g_{i j}(t, \mathbf{x})=\mathrm{e}^{2 \sigma(t, \mathbf{x})} \tilde{g}_{i j}(\mathbf{x}) .
$$

Recall now that (55) and (60) are Weyl covariant. Performing a Weyl rescaling with $\mathcal{B}(t, \mathbf{x})=\mathrm{e}^{2 \sigma(t, \mathbf{x})}$ removes the time dependence from the metric, while it transforms the other fields as

$\tilde{\Omega}(t, \mathbf{x})=\mathrm{e}^{-z \sigma(t, \mathbf{x})} \Omega(t, \mathbf{x})$,

$\tilde{\varphi}_{i}(t, \mathbf{x})=\varphi_{i}(t, \mathbf{x})-z\left(\partial_{i}+b_{i}(t, \mathbf{x}) \partial_{t}\right) \sigma(t, \mathbf{x}), \quad \tilde{\theta}(t, \mathbf{x})=0$.

The Killing field is invariant, $\tilde{\xi}=\xi=\tilde{f} \tilde{E}+Y^{i} E_{i}$ with $\tilde{E}=\mathrm{e}^{z \sigma} E$, and this leads to

$$
\begin{aligned}
\tilde{f}(t, \mathbf{x}) & =\mathrm{e}^{-z \sigma(t, \mathbf{x})} f(t, \mathbf{x}), \quad \tilde{Y}^{i}(\mathbf{x})=Y^{i}(\mathbf{x}), \\
\tilde{Y}_{i}(\mathbf{x}) & =\tilde{g}_{i j}(\mathbf{x}) Y^{j}(\mathbf{x}) .
\end{aligned}
$$

Equations (55) and (60) finally become equations for $\tilde{f}(t, \mathbf{x})$ and $Y^{i}(\mathbf{x})$,

$$
\begin{aligned}
& \tilde{\nabla}_{i} Y_{j}+\tilde{\nabla}_{j} Y_{i}=\frac{2}{d} \tilde{\nabla}_{k} Y^{k} \tilde{g}_{i j}, \\
& \tilde{\Omega}^{-1} \partial_{t} \tilde{f}=\frac{z}{d} \tilde{\nabla}_{k} Y^{k}-\tilde{\varphi}_{k} Y^{k},
\end{aligned}
$$

where $\tilde{\nabla}_{i}$ is the Levi-Civita connection for $\tilde{g}_{i j}$.

The first equation is an ordinary conformal Killing equation, and its solutions $\left\{Y^{i}(\mathbf{x})\right\}$ are the generators of the conformal group for $\mathcal{S}$ equipped with a metric $\tilde{g}_{i j}(\mathbf{x})$. Given any such vector in $H$ solving (64),

$$
\bar{\xi}_{Y}=Y^{i}(\mathbf{x}) E_{i}=Y^{i}(\mathbf{x})\left(\partial_{i}+b_{i}(t, \mathbf{x}) \partial_{t}\right)
$$

\footnotetext{
${ }^{9}$ The left-hand side of Eq. (60) can actually be recast using Weyl-covariant derivatives, based on the Weyl connection $\boldsymbol{A}=\frac{1}{z} \boldsymbol{\varphi}+\frac{1}{d} \theta \boldsymbol{e}$, which transforms as $\boldsymbol{A} \mapsto \boldsymbol{A}-\mathrm{d} \ln \mathcal{B}$.
} 
(the subscript $Y$ stresses that the vector field at hand depends on the set $\left\{Y^{i}(\mathbf{x})\right\}$ ), Eq. (65) provides a solution for $\tilde{f}(t, \mathbf{x})$,

$$
\begin{aligned}
\tilde{f}(t, \mathbf{x})= & T(\mathbf{x})+\frac{z}{d} \int^{t} \mathrm{~d} t^{*} \tilde{\Omega}\left(t^{*}, \mathbf{x}\right) \\
& \times\left(\tilde{\nabla}_{i} Y^{i}(\mathbf{x})-\frac{d}{z} \tilde{\varphi}_{i}\left(t^{*}, \mathbf{x}\right) Y^{i}(\mathbf{x})\right) .
\end{aligned}
$$

Here $T(\mathbf{x})$ is an arbitrary smooth function of weight $-z$, which specifies any conformal Carrollian Killing field.

Before we further investigate this family of conformal Carrollian Killing vectors, we should pause and make contact with previous results reached in the already quoted literature. The situation that has been studied in [4] corresponds in our language to $\sigma=0$ and $\Omega=1$. This means in particular that the metric is time independent. In Ref. [4] no Ehresmann connection was introduced. We could therefore set it to 0 , or better leave $b_{i}(t, \mathbf{x})$ unspecified, because, as mentioned earlier for a time-independent metric, it is not expected to play any role in the conformal algebra. Indeed, using (64) we find the precise family of vectors $\bar{\xi}_{Y}$ as in (66), which combined with (67) leads to

$$
\xi_{T, Y}=\left(T(\mathbf{x})+\frac{z}{d} t \tilde{\nabla}_{i} Y^{i}(\mathbf{x})\right) \partial_{t}+Y^{i}(\mathbf{x}) \partial_{i}
$$

irrespective of $b_{i}(t, \mathbf{x})$ (again the subscript $T, Y$ recalls the dependence on $\left.\left\{T(\mathbf{x}), Y^{i}(\mathbf{x})\right\}\right)$. Therefore the corresponding algebra is infinite dimensional and emerges as the semidirect product of the conformal group of $g=\tilde{g}(\mathbf{x})$ on $\mathcal{S}$, generated by $Y^{i}(\mathbf{x}) \partial_{i}$, with supertranslations. For a flat or conformally flat metric on $\mathcal{S}$, the spatial conformal algebra in $d$ dimensions is $\mathfrak{g} \mathfrak{v}(d+1,1)$, and the conformal Carrollian Killing fields (68) $\operatorname{span}^{10} \mathfrak{c c a r r}_{N}(d+1)=\mathfrak{g} \mathfrak{o}(d+1,1) \ltimes \mathfrak{I}_{N}$, where $z=2 / N$. The standard conformal Carrollian algebra $\mathfrak{c c a r r}(d+1)$ refers to dynamical exponent $z=1$ (level $N=2): \quad \mathfrak{c} a \mathfrak{r} r(d+1)=\mathfrak{c c a r r}_{2}(d+1)$. This algebra emerges as the null-infinity isometry algebra of asymptotically flat $d+2$-dimensional spacetimes in Bondi gauge, $\mathfrak{b} \mathfrak{m} \mathfrak{z}(d+2) .{ }^{11}$

Our general analysis embraces the above case, by including time dependence in the spatial metric $g$ and a general scale factor $\Omega(t, \mathbf{x})$ on top of the Ehresmann connection $b_{i}(t, \mathbf{x})$. Despite these generalizations, as a

\footnotetext{
${ }^{10}$ This algebra is defined in the literature for integer $N$.

${ }^{11}$ As before, strictly speaking this is valid for $d=1$ and 2 (where furthermore $\tilde{g}$ is always conformally flat). For higher $d$, it was presumed to hold by some authors [3]. However, gauge conditions exist for the Bondi-gauge null-infinity behavior of asymptotically flat spacetimes that render $\mathfrak{b} \mathfrak{m} \mathfrak{s}(d+2)$ finite dimensional [22], and with this choice $\mathfrak{c} \mathfrak{a} \mathfrak{r r}_{2}(d+1) \neq \mathfrak{b} \mathfrak{m} \mathfrak{g}(d+2)$. This does not exclude that less restrictive gauge fixing might be considered leading to other, possibly infinite-dimensional $\mathfrak{b} \mathfrak{m} \mathfrak{g}(d+2)$ algebras for $d \geq 3$.
}

direct consequence of the factorized time dependence in the metric [see (61)] due to the requirement of vanishing shear, the structure of the conformal Carrollian Killing vectors remains unaltered, i.e., as in (68): their algebra is the semidirect product of the conformal group of $\tilde{g}(\mathbf{x})$ on $\mathcal{S}$ with supertranslations at dynamical exponent $z$. This statement is shown as follows.

Using (67), we obtain the general conformal Carrollian Killings as vector fields in $T \mathcal{C}$,

$$
\begin{aligned}
\xi_{T, Y}= & \left(T(\mathbf{x})+\frac{z}{d} \int^{t} \mathrm{~d} t^{*} \tilde{\Omega}\left(t^{*}, \mathbf{x}\right)\right. \\
& \left.\times\left(\tilde{\nabla}_{i} Y^{i}(\mathbf{x})-\frac{d}{z} \tilde{\varphi}_{i}\left(t^{*}, \mathbf{x}\right) Y^{i}(\mathbf{x})\right)\right) \tilde{E}+Y^{i}(\mathbf{x}) E_{i} .
\end{aligned}
$$

We can unravel the structure of these conformal Carroll Killings and of their algebra by introducing an invariant local clock,

$$
C(t, \mathbf{x}) \equiv \int^{t} \mathrm{~d} t^{*} \tilde{\Omega}\left(t^{*}, \mathbf{x}\right)
$$

This in fact is a specific instance of $C_{\gamma}=\int_{\gamma} \tilde{\Omega}(\mathrm{d} t-b)$ with $\gamma$ being a path in $\mathcal{C}$. In (70), $C(t, \mathbf{x})$ appears as a local function because the path runs along a vertical fiber starting at, say, the zero section reference to which we have suppressed. ${ }^{12}$ Using (19) and (70) we reach the following identity:

$$
\int{ }^{t} \mathrm{~d} t^{*} \tilde{\Omega}\left(t^{*}, \mathbf{x}\right) \tilde{\varphi}_{i}\left(t^{*}, \mathbf{x}\right)=E_{i}(C(t, \mathbf{x})),
$$

which enables us to express (69) as

$$
\begin{aligned}
\xi_{T, Y}= & \left(T(\mathbf{x})-Y^{i} E_{i}(C(t, \mathbf{x}))+\frac{z}{d} C(t, \mathbf{x}) \tilde{\nabla}_{i} Y^{i}(\mathbf{x})\right) \\
& \times \tilde{E}+Y^{i}(\mathbf{x}) E_{i} .
\end{aligned}
$$

The invariant clock defines a Carrollian diffeomorphism [see (5)] with $t^{\prime}=C(t, \mathbf{x})$ and $\mathbf{x}^{\prime}=\mathbf{x}$. Under this diffeomorphism $\tilde{\Omega} \rightarrow 1, \tilde{E} \rightarrow \partial_{t^{\prime}}$, while (72) reads now precisely as (68) with $t$ traded for $t^{\prime}$. This demonstrates the earlier statement about the algebra of conformal Carrollian Killing vectors of a shearless Carroll spacetime.

Summarizing, shearless Carrollian spacetimes, i.e., spacetimes equipped with a metric of the form $g_{i j}(t, \mathbf{x})=$ $\mathrm{e}^{2 \sigma(t, \mathbf{x})} \tilde{g}_{i j}(\mathbf{x})$, have a conformal isometry algebra that depends only on $\tilde{g}(\mathbf{x}), d$ and $z$ : it is the semidirect product of the conformal algebra of $\mathcal{S}$ equipped with $\tilde{g}(\mathbf{x})$ and supertranslations at level $N=2 / z$. This conclusion is valid

\footnotetext{
${ }^{12}$ We refer to $C(t, \mathbf{x})$ as an invariant local clock because it defines an integration measure on each one-dimensional fiber, a proper time.
} 
irrespective of $\Omega(t, \mathbf{x})$ and $b_{i}(t, \mathbf{x})$. On the one hand, $\Omega(t, \mathbf{x})$ can disappear from the expression (72) of the Killings upon an appropriate Carrollian diffeomorphism driven by the invariant local clock. Hence its presence does not affect the algebra. On the other hand, although the Ehresmann connection $b_{i}(t, \mathbf{x})$ cannot be removed with Carrollian diffeomorphisms (unless its field strength $\varpi$ and acceleration $\varphi$ vanish), it cancels out between the last two terms in (72). This is not insignificant though, and we discuss it in the remainder of the present chapter.

The set of vectors $Y=Y^{i}(\mathbf{x}) \partial_{i} \in T \mathcal{S}$ with $\left\{Y^{i}(\mathbf{x})\right\}$ solving (64) realizes the conformal algebra of $\tilde{g}$,

$$
\left[Y, Y^{\prime}\right]=\left[Y^{i} \partial_{i}, Y^{\prime j} \partial_{j}\right]=Y^{\prime \prime k} \partial_{k}=Y^{\prime \prime},
$$

with

$$
Y^{\prime \prime k}=Y^{i} \partial_{i}\left(Y^{\prime k}\right)-Y^{\prime i} \partial_{i}\left(Y^{k}\right)
$$

These vectors act generally on functions $\phi(\mathbf{x})$. One may instead contemplate a realization in terms of Carrollian vectors $\bar{\xi}_{Y} \in H$ as in (66) acting on functions $\Phi(t, \mathbf{x})$ of $\mathcal{C}$. In this case,

$$
\begin{aligned}
{\left[\bar{\xi}_{Y}, \bar{\xi}_{Y^{\prime}}\right] } & =\bar{\xi}_{\left[Y, Y^{\prime}\right]}-\boldsymbol{\varpi}\left(Y, Y^{\prime}\right) E \\
& =\bar{\xi}_{\left[Y, Y^{\prime}\right]}-\tilde{\boldsymbol{\varpi}}\left(Y, Y^{\prime}\right) \tilde{E} \in V \oplus H,
\end{aligned}
$$

where $\varpi\left(Y, Y^{\prime}\right)=\varpi_{i j} Y^{i} Y^{\prime j}$ and $\tilde{\boldsymbol{\varpi}}=\mathrm{e}^{-z \sigma} \varpi$. Because of the Ehresmann connection, this realization is not faithfully the conformal algebra (73) of $\tilde{g}$, except if the Carrollian torsion is 0 (horizontal piece of the Ehresmann curvature), which coincides with the condition for $H$ to be integrable ${ }^{13}$ (or if the action is limited to functions of $\mathbf{x}$ only, which is not what we want). Furthermore the extra $V$-term is not a central extension, unless the Carrollian acceleration vanishes (in this case $E$ and $E_{i}$ commute).

The expression in parentheses present in (72) suggests defining, for each set $\left\{Y^{i}(\mathbf{x})\right\}$ associated with a solution of (64), a Carrollian operator $M_{Y}$ acting on any function $\Phi(t, \mathbf{x})$ of $\mathcal{C}$ as

$$
M_{Y}(\Phi) \equiv Y^{i} E_{i}(\Phi)-\frac{z}{d} \Phi \tilde{\nabla}_{i} Y^{i}
$$

The mapping $Y \rightarrow M_{Y}$ is a representation of the group of conformal Killing vectors of $\tilde{g}$, which however is again not faithful as the commutator exhibits an extra term, similar to the one in (75), possibly vanishing in the same circumstances,

\footnotetext{
${ }^{13}$ Generally, one expects invariants that prevent the horizontal part of the Ehresmann connection from being flat. For example, in $d=2$, one might have nonzero Chern class $c=\frac{1}{2 \pi} \int_{\mathcal{S}} \varpi$.
}

$$
\begin{aligned}
{\left[M_{Y}, M_{Y^{\prime}}\right](\Phi) } & \equiv M_{Y}\left(M_{Y^{\prime}}(\Phi)\right)-M_{Y^{\prime}}\left(M_{Y}(\Phi)\right) \\
& =M_{\left[Y, Y^{\prime}\right]}(\Phi)-\tilde{\boldsymbol{\varpi}}\left(Y, Y^{\prime}\right) \tilde{E}(\Phi) .
\end{aligned}
$$

Using now the map (76) and $\bar{\xi}_{Y} \in H$ given in Eq. (66), the conformal Killing field in $T \mathcal{C}$, Eq. (72), is recast as

$$
\xi_{T, Y}=\left(T(\mathbf{x})-M_{Y}(C)(t, \mathbf{x})\right) \tilde{E}+\bar{\xi}_{Y}
$$

For vanishing $T(\mathbf{x})$, the representation $M_{Y}$ defines a lift of $\bar{\xi}_{Y}=Y^{i} E_{i} \in H \rightarrow T \mathcal{C}$ through the map

$$
\bar{\xi}_{Y} \mapsto \xi_{0, Y}=\bar{\xi}_{Y}-M_{Y}(C) \tilde{E} .
$$

This lift provides a faithful and Carrollian (i.e., acting on functions of $t$ and $\mathbf{x}$ ) realization of the conformal isometry algebra (73) of $\tilde{g}$ on $T \mathcal{C}$, thanks to the cancellation of the extra term appearing in (75) and (77). Even though the Ehresmann connection does not appear ultimately in the conformal algebra, when nonvanishing, it adjusts for making compatible the realization of the algebra with Carrollian diffeomorphism invariance. This is yet another of its numerous facets. For nonvanishing $T(\mathbf{x})$, we obtain the following commutation relations for the complete conformal Carrollian Killing fields $(78)^{14}$ :

$$
\left[\xi_{T, Y}, \xi_{T^{\prime}, Y^{\prime}}\right]=\xi_{M_{Y}\left(T^{\prime}\right)-M_{Y^{\prime}}(T),\left[Y, Y^{\prime}\right]} .
$$

This is the usual pattern for conformal Carrollian and BMS algebras.

\section{CONCLUSIONS}

In this work, we have considered Carrollian geometries from various perspectives: their defining properties, their emergence on embedded null hypersurfaces, and their conformal symmetries. We have emphasized the interpretation of Carrollian spacetime as a fiber bundle endowed with an Ehresmann connection. Realized by a one-form field, this connection defines the splitting of the tangent bundle into vertical and horizontal components. The vertical component coincides precisely with the kernel of a degenerate metric, which is the last piece of equipment for a Carroll structure. It is worth stressing that all defining fields (Ehresmann connection, metric and scale factor) have been assumed space and time dependent throughout the paper.

The vertical versus horizontal canonical separation is preserved by the subset of Carrollian diffeomorphisms. These enable the reduction of spacetime tensors into purely spatial components, the paradigm being Carrollian torsion and acceleration, emerging as reduced components of the Ehresmann curvature. Other geometric objects can be introduced using the degenerate metric, such as shear and expansion, and even further based on a horizontal

\footnotetext{
${ }^{14}$ We use here the identity $\tilde{E}\left(M_{Y}(C)\right)=\tilde{\varphi}_{i} Y^{i}-\frac{z}{d} \tilde{\nabla}_{i} Y^{i}$.
} 
connection, which we only alluded to when discussing the Christoffel-Carroll symbols. Investigating the types of connections that can be defined on the full tangent bundle $T C$ is an interesting subject that has been touched upon in the literature, ${ }^{15}$ but, we believe, deserves more attention, e.g., in the case of Weyl connections.

The above ingredients (Ehresmann connection and vertical and horizontal sub-bundles) arise naturally on null hypersurfaces embedded in Lorentzian spacetimes, and specific tensors such as Carrollian shear, acceleration and expansion are inherited from the ambient geometry. Our analysis was here confined to the instance of genuine null foliations, but can be adapted to the case of boundary null hypersurfaces, such as black-hole horizons or null infinities.

The last element of our investigation concerns symmetries, and more specifically conformal isometries of Carrollian spacetimes. Contrary to pseudo-Riemannian geometries, the definition of (conformal) isometries cannot rely solely on the Killing equation for the metric, because the latter is degenerate. Here we complied with the standard definition of the conformal Carrollian Killing vectors, and additionally restricted our analysis to the case of shearless Carrollian structures. Although seemingly innocuous, as time dependence remains general both in the scale factor and in the Ehresmann connection, this limitation is quite severe. Indeed time dependence of the metric is factorized and this ultimately drives us to the standard semidirect product of the conformal isometry algebra of the metric with supertranslations. This is infinite dimensional and coincides with $\mathfrak{c c a r r}_{N}(d+1)$, for conformally flat spatial metrics. One thus recovers $\mathfrak{b} \mathfrak{m} \mathfrak{g}(d+2)$ in $d=1$ and 2 , and possibly in a higher dimension with some appropriate definition of the BMS algebra. Our study has the virtue of

\footnotetext{
${ }^{15}$ See Appendix A of [16] for a classification of compatible connections, i.e., connections that parallel transport the degenerate metric $g$ and the vector field $E$.
}

sustaining the robustness of the format already known to emerge in static Carrollian spacetimes without scale factor or Ehresmann connection. It stresses the role of the shear, but leaves open the probe of the conformal Carrollian isometries, when the latter is nonzero. It also illustrates another subtle role of the Ehresmann connection, which allows one to lift without alteration the conformal isometry algebra of the metric from the basis tangent bundle $T \mathcal{S}$ to the Carrollian tangent bundle $T C$.

Although relatively confined, our investigation touches upon several timely and perhaps deep issues. Conformal symmetries and in particular the BMS algebra are known to appear as the backbone of conserved charges in asymptotically flat spacetimes. In addition, the role of null hypersurfaces has been appreciated in flat holography, where they are expected to replace the timelike foliations relevant in anti-de Sitter holography. In particular, their symplectic structure should play a significant role in giving an alternative reading of the gravitational degrees of freedom. Clearly, Carrollian spacetimes and their symmetries are the central concepts in all these developments, which deserve further analysis, possibly in the lines of our current work.

\section{ACKNOWLEDGMENTS}

We thank Glenn Barnich, Laurent Freidel, Kevin Morand, and Tassos Petkou for fruitful discussions. This research was supported in part by the ANR-16-CE31-0004 contract Black-dS-String, by Perimeter Institute for Theoretical Physics, and by the US Department of Energy under Contract No. DE-SC0015655. Research at Perimeter Institute is supported by the Government of Canada through the Department of Innovation, Science, and Economic Development Canada and by the Province of Ontario through the Ministry of Research, Innovation, and Science. The Ecole Polytechnique preprint number is CPHT-RR010.022019.
[1] J.-M. Lévy-Leblond, Une nouvelle limite nonrelativiste du groupe de Poincaré, Ann. Inst. Henri Poincaré 3, 1 (1965).

[2] C. Duval, G. W. Gibbons, P. A. Horvathy, and P. M. Zhang, Carroll versus Newton and Galilei: Two dual nonEinsteinian concepts of time, Classical Quantum Gravity 31, 085016 (2014).

[3] C. Duval, G. W. Gibbons, and P. A. Horvathy, Conformal Carroll groups and BMS symmetry, Classical Quantum Gravity 31, 092001 (2014).

[4] C. Duval, G. W. Gibbons, and P. A. Horvathy, Conformal Carroll groups, J. Phys. A 47, 335204 (2014).
[5] M. Henneaux, Geometry of zero-signature space-times, Bull. Soc. Math. Belg. 31, 47 (1979).

[6] C. Duval, G. W. Gibbons, and P. Horvathy, Celestial mechanics, conformal structures and gravitational waves, Phys. Rev. D 43, 3907 (1991).

[7] J. Hartong, Gauging the Carroll algebra and ultra-relativistic gravity, J. High Energy Phys. 08 (2015) 069.

[8] K. Morand, Embedding Galilean and Carrollian geometries I. Gravitational waves, arXiv:1811.12681.

[9] G. Barnich and C. Troessaert, Aspects of the BMS/CFT correspondence, J. High Energy Phys. 05 (2010) 062. 
[10] A. Bagchi, The BMS/GCA Correspondence, Phys. Rev. Lett. 105, 171601 (2010).

[11] H. Bondi, M. G. van der Burg, and A. W. Metzner, Gravitational waves in general relativity, VII. Waves from axi-symmetric isolated systems, Proc. R. Soc. A 269, 21 (1962).

[12] R. K. Sachs, Asymptotic symmetries in gravitational theories, Phys. Rev. 128, 2851 (1962).

[13] G. Barnich and C. Troessaert, Symmetries of Asymptotically Flat 4-Dimensional Spacetimes at Null Infinity Revisited, Phys. Rev. Lett. 105, 111103 (2010).

[14] A. Ashtekar, Geometry and physics of null infinity, in One Hundred Years of General Relativity, edited by L. Bieri and S. T. Yau (International press, Boston, 2015), p. 99.

[15] X. Bekaert and K. Morand, Connections and dynamical trajectories in generalised Newton-Cartan gravity I. An intrinsic view, J. Math. Phys. (N.Y.) 57, 022507 (2016).

[16] X. Bekaert and K. Morand, Connections and dynamical trajectories in generalised Newton-Cartan gravity II.
An ambient perspective, J. Math. Phys. (N.Y.) 59, 072503 (2018).

[17] L. Ciambelli, C. Marteau, A. C. Petkou, P. M. Petropoulos, and K. Siampos, Covariant Galiliean versus Carrollian hydrodynamics from relativistic fluids, Classical Quantum Gravity 35, 165001 (2018).

[18] L. Ciambelli, C. Marteau, A. C. Petkou, P. M. Petropoulos, and K. Siampos, Flat holography and Carrollian fluids, J. High Energy Phys. 07 (2018) 165.

[19] V. Chandrasekaran, É. É. Flanagan, and K. Prabhu, Symmetries and charges of general relativity at null boundaries, J. High Energy Phys. 11 (2018) 125.

[20] A. J. Speranza, Geometrical tools for embedding fields, submanifolds, and foliations, arXiv:1904.08012.

[21] F. Hopfmüller and L. Freidel, Gravity degrees of freedom on a null surface, Phys. Rev. D 95, 104006 (2017).

[22] A. Campoleoni, D. Francia, and C. Heissenberg, Asymptotic charges at null infinity in any dimension, Universe 4, 47 (2018). 